\title{
Non-Hermitian phase transition and eigenstate localization induced by asymmetric coupling
}

\author{
P. Wang, L. Jin, ${ }^{*}$ and Z. Song ${ }^{\dagger}$ \\ School of Physics, Nankai University, Tianjin 300071, China
}

(Received 5 March 2019; revised manuscript received 14 May 2019; published 19 June 2019)

\begin{abstract}
We investigate a uniformly coupled non-Hermitian system with asymmetric coupling amplitude. The asymmetric coupling equals to a symmetric coupling threaded by an imaginary gauge field. In a closed configuration, the imaginary gauge field leads to an imaginary magnetic flux, which induces a non-Hermitian phase transition. For an open boundary, the imaginary gauge field results in an eigenstate localization. The eigenstates under Dirac and biorthogonal norms and the scaling laws are quantitatively investigated to show the affect of asymmetric coupling induced one-way amplification. However, the imaginary magnetic flux does not inevitably induce the non-Hermitian phase transition for systems without translation invariance, which is elucidated from the non-Hermitian phase transition in the non-Hermitian ring with a single coupling defect. Our findings provide insights into the non-Hermitian phase transition and one-way localization.
\end{abstract}

DOI: 10.1103/PhysRevA.99.062112

\section{INTRODUCTION}

The parity-time $(\mathcal{P} \mathcal{T})$ symmetry confinement enables a purely real spectrum of the non-Hermitian system [1-8]. In $\mathcal{P} \mathcal{T}$-symmetric non-Hermitian systems, the degree of nonHermiticity determines a phase transition [9] at the exceptional points [10-19], where the system is defective and the intensity polynomial increases [20]. Exceptional points are valuable in quantum metrology and sensing [21-23]. The intensity of initial excitation oscillates in the exact $\mathcal{P} \mathcal{T}$ symmetric phase with purely real eigenvalues [24-26]. In the broken $\mathcal{P} \mathcal{T}$-symmetric phase, the eigenvalues become conjugation pairs and the intensity exponentially increases. Non-Hermitian systems exhibit many intriguing dynamical phenomena due to the nonorthogonality of the eigenmodes, such as coherent perfect absorption [27], unidirectional invisibility or unidirectional reflectionless [28,29], unidirectional perfect absorption [30], unidirectional propagation [31], and unidirectional lasing [31-33]. The nonlinear non-Hermitian systems with asymmetric structures exhibit intriguing nonreciprocal properties [34].

The $\mathcal{P} \mathcal{T}$-symmetric gain and loss are experimentally realized in atomic systems [35], optical waveguides [26,29,36-38], resonators [39-41], photonic crystals [42,43], electronic circuits [44], and acoustics systems [45,46]. By employing gain, optical isolation [47], single mode lasing in $\mathcal{P} \mathcal{T}$ symmetric coupled resonators [48], unidirectional lasing due to asymmetric backscattering [33], and topological lasing [49] have been experimentally demonstrated. Other non-Hermitian

\footnotetext{
*jinliang@nankai.edu.cn

†songtc@nankai.edu.cn
}

Published by the American Physical Society under the terms of the Creative Commons Attribution 4.0 International license. Further distribution of this work must maintain attribution to the author(s) and the published article's title, journal citation, and DOI. elements include pure imaginary coupling and asymmetric coupling. The pure imaginary coupling $i \gamma$ has been proposed in atomic systems to demonstrate anti- $\mathcal{P} \mathcal{T}$ symmetry [35] . The asymmetric coupling of directional amplification or attenuation [50,51] has been realized in coupled resonators, or atomic gases [52]. Recently, non-Hermitian topological systems have received extensive attention [53-81], in particular, for systems with asymmetric coupling. The asymmetric coupling induces all the eigenstates localized at the system boundary, which is called the non-Hermitian skin effect [82-89]. The skin effect and edge mode in the non-Hermitian system are revealed using the Green's-function method [79]. The non-Hermitian Su-Schrieffer-Heeger models with stagger asymmetric coupling with and without chiral-inversion symmetry are investigated in Ref. [84]. The nature of nonHermiticity appearance is revealed to be critical. The oneway amplification or attenuation induces the nonzero nonHermitian Aharonov-Bohm (AB) effect and the skin effect. The chiral-inversion symmetry protects the bulk-boundary correspondence in the non-Hermitian topological systems, and prevents the non-Hermitian skin effect. In non-Hermitian topological systems, the non-Hermitian $\mathrm{AB}$ effect induces the non-Hermitian phase transition and topological phase transition [82-86]; however, whether the non-Hermitian AB effect induces non-Hermitian phase transition in the non-Hermitian system in general cases is an interesting question.

In this paper, we investigate the non-Hermitian phase transition induced by asymmetric coupling amplitude. The asymmetric coupling effectively generates a gauge invariant imaginary field; the imaginary gauge field in a closed area induces an imaginary magnetic flux, which does not inevitably result in a non-Hermitian phase transition, in particular, for the non-Hermitian systems that are not translation invariant. This is elucidated through a non-Hermitian nonuniform ring system with single defective coupling. The situation dramatically differs from that in the translation invariant topological systems, where the imaginary gauge field enters the wave vector. The momentum becomes a complex number and 
induces the non-Hermitian phase transition. The nonHermitian skin effect found in topological systems has renewed the research interest for the localization effect. We consider a uniform chain to quantitatively investigate the nonHermitian localization without the influence of system topology. We concentrate on capturing the properties of asymmetric coupling induced eigenstate localization; alternatively, the localization is a unidirectional amplification. Therefore, the averaged inverse participation ratio (IPR) under the definition of the Dirac norm is investigated. At weak non-Hermiticity, the averaged IPR is inversely proportional to the system size when the system size is small; at large non-Hermiticity, the eigenstates are tightly localized at the system boundary and the IPR is insensitive to the system size. In contrast, the gauge invariant field does not affect the biorthogonal norm of the eigenstates; the biorthogonal IPR is inversely proportional to the system size like the extended states although the Dirac probability distributions are localized. Our findings provide insights for the non-Hermitian asymmetric coupling, nonHermitian skin effect, and non-Hermitian phase transition.

The paper is organized as follows. In Sec. II, we introduce the non-Hermitian system with asymmetric coupling. In Sec. III, we exactly solve the system and show its energy spectrum. In Sec. IV, we study a nonuniform case with single coupling difference. In Sec. V, the eigenstates of the system under open boundary condition are investigated, the scaling law of the eigenstates is presented. In Sec. VI, the biorthogonal IPR of the eigenstates is discussed. Our results are summarized in Sec. VII.

\section{MODEL}

Before introducing the non-Hermitian system, we first consider a discrete one-dimensional tight-binding lattice system with uniform coupling strength. The coupling amplitude has a Peierls phase factor $e^{i \varphi}$ in the front, and the system Hamiltonian reads

$$
H_{0}=\kappa \sum_{j=1}^{N}\left(e^{i \varphi} a_{j}^{\dagger} a_{j+1}+\text { H.c. }\right),
$$

where $a_{j}^{\dagger}\left(a_{j}\right)$ is the creation (annihilation) operator of site $j$. The tight-binding system is schematically illustrated in Fig.1(a) in a closed ring configuration under periodical boundary condition $a_{j}=a_{j+N}$ and $a_{j}^{\dagger}=a_{j+N}^{\dagger}$ for total system size $N$. The Peierls phase factor $e^{i \varphi}$ in the couplings induces a magnetic flux $N \varphi$ in the $N$-site ring system. The dispersion relation is given by

$$
E=2 \kappa \cos (k+\varphi)
$$

where the wave vector $k$ in the momentum space is discrete, being $k=2 n \pi / N$ with integer $n=1, \ldots, N$. The corresponding eigenstate for $E$ is a plane wave with momentum $k$ in the form of $\left|\psi_{k}\right\rangle=\sqrt{1 / N} \sum_{j=1}^{N} e^{i k j} a_{j}^{\dagger}|\mathrm{vac}\rangle$. Under the influence of the magnetic flux $N \varphi$, the momentum effectively shifts by a value $\varphi$; however, the dispersion relation remains unchanged. The energy spectrum of the uniform ring under the influence of magnetic flux is depicted in Fig. 1(b) for an $N=8$ system. Eight energy levels are all changed to (a)

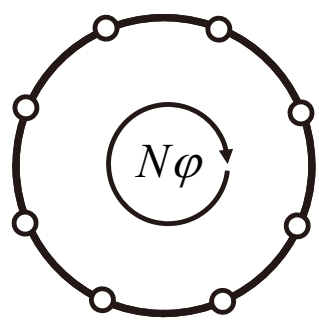

(c)
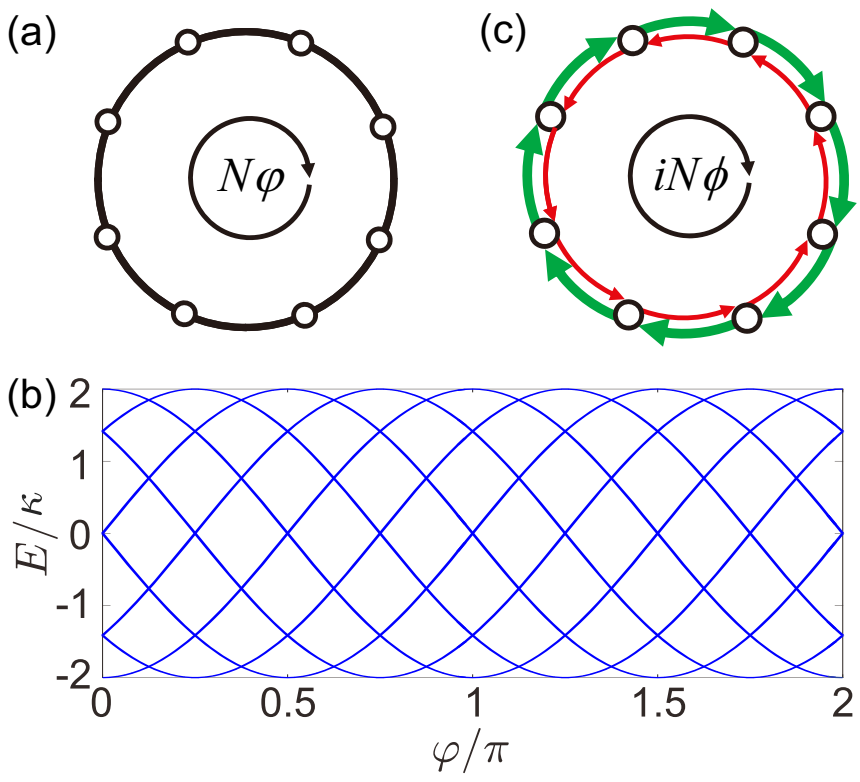

FIG. 1. (a) Schematic of the uniform ring enclosed with magnetic flux $N \varphi$. (b) Energy spectrum of a uniform ring under the influence of magnetic flux. The system size is chosen as $N=8$ and the parameter is $\kappa=1$ in the plot. (c) Schematic of the uniform ring with asymmetric coupling strength. The green (red) arrow indicates the tunneling in the clockwise (counterclockwise) direction. The asymmetric coupling mimics a gauge field, which induces the imaginary magnetic flux $-i N \phi$.

their opposite energies after their momentum shifted by $\pi$ at $\varphi=\pi$.

In Fig. 1(c), the uniform tight-binding lattice with nonHermitian asymmetric coupling is shown. The asymmetric coupling strength indicates the different amplitudes when particles or photons tunnel in opposite directions. The Hamiltonian reads $H=\sum_{j=1}^{N}\left(\alpha a_{j}^{\dagger} a_{j+1}+\beta a_{j+1}^{\dagger} a_{j}\right)$. Considering real $\alpha, \beta>0$ without loss of generality, the coupling amplitudes can be rewritten as $\alpha=\sqrt{\alpha \beta} \sqrt{\alpha / \beta}$ and $\beta=\sqrt{\alpha \beta} \sqrt{\beta / \alpha}$. The Hamiltonian reduces into another form of

$$
H=\sqrt{\alpha \beta} \sum_{j=1}^{N}\left(e^{\phi} a_{j}^{\dagger} a_{j+1}+e^{-\phi} a_{j+1}^{\dagger} a_{j}\right),
$$

where $e^{\phi}=\sqrt{\alpha / \beta}$; the Hamiltonian $H$ has a uniform symmetric coupling, but threaded by an imaginary magnetic flux [84]. The nonreciprocal amplification or attenuation factor accumulates when particles or photons circle along one direction in the ring configuration; the factor accumulated after circling one round gives $e^{ \pm N \phi}$ in opposite direction. The accumulation can be understood from the viewpoint of an imaginary gauge field and imaginary magnetic flux, which are both gauge invariant under gauge transformations [50,51]. The coupled ring resonator array is a physical realization of $H$ [50], where the main resonators are evanescently coupled through auxiliary resonators. To introduce effective asymmetric coupling between the main resonators, the auxiliary ring resonators have half-perimeter gain and half-perimeter loss. Photons tunneling between the main resonators are thus direction dependent, amplified in one direction and attenuated in the opposite 
direction; this results in the asymmetric coupling in $H$. In the coupled resonator array, the light intensity distribution of eigenstate reflects the localization effect.

Setting $\bar{b}_{j}=e^{-j \phi} a_{j}^{\dagger}, b_{j}=e^{j \phi} a_{j}$, the operators satisfy the commutation relation $\left[b_{l}, \bar{b}_{j}\right]=\left[a_{l}, a_{j}^{\dagger}\right]=\delta_{l j}$. We obtain a uniform ring Hamiltonian with symmetric coupling strength $\sqrt{\alpha \beta}$ except one asymmetric coupling at the connection between the head and tail. The only asymmetric coupling is $\sqrt{\alpha \beta} e^{ \pm N \phi}$ associated with the amplification or attenuation factor $e^{ \pm N \phi}$. The non-Hermitian Hamiltonian is in the form of $H=\sqrt{\alpha \beta}\left[\sum_{j=1}^{N-1}\left(\bar{b}_{j} b_{j+1}+\bar{b}_{j+1} b_{j}\right)+e^{i \Phi} \bar{b}_{N} b_{1}+\right.$ $\left.e^{-i \Phi} \bar{b}_{1} b_{N}\right]$, where the amplification or attenuation factor is given by $e^{i \Phi}=(\sqrt{\alpha / \beta})^{N}$, which yields an effective imaginary magnetic flux $\Phi=-i N \phi$ threaded in the ring configuration. A particle or photon circling one round in the ring accumulates an additional amplification (attenuation) factor $e^{i \Phi}\left(e^{-i \Phi}\right)$ on its wave function in the clockwise (counterclockwise) direction.

\section{ENERGY SPECTRUM}

The eigenvalues of the non-Hermitian system are obtained after applying a Fourier transformation:

$$
a_{k}^{\dagger}=\frac{1}{\sqrt{N}} \sum_{j=1}^{N} e^{i k j} a_{j}^{\dagger},
$$

where $k=2 \pi n / N$ with integer $n=1, \ldots, N$. The Hamiltonian $H$ in the momentum space is given by

$$
H=\sqrt{\alpha \beta} \sum_{k}\left[e^{i k+\phi}+e^{-(i k+\phi)}\right] a_{k}^{\dagger} a_{k} .
$$

Then, the eigenvalues of $H$ are given by

$$
E=2 \sqrt{\alpha \beta} \cos (k-i \phi)
$$

The energy spectrum of the non-Hermitian lattice with an imaginary magnetic flux has an identical form of expression in comparison with the Hermitian system threaded by a real magnetic flux. In contrast to the real magnetic field that shifts the wave vector $k$ in the momentum space, the imaginary magnetic field results in the wave vector extended into the complex plane. This induces a non-Hermitian phase transition. The Aharonov-Bohm effect occurs only if the gauge field is enclosed in the ring. In an open chain, the eigenvalues are $E=2 \sqrt{\alpha \beta} \cos [n \pi /(N+1)]$, not affected by the imaginary gauge field.

The energy spectrum of the non-Hermitian lattice [Fig. 1(c)] is depicted in Fig. 2 as a function of the degree of coupling asymmetry. Under periodical boundary condition, the asymmetry in the coupling $(\alpha \neq \beta)$ leads to a complex spectrum in Figs. 2(a)-2(c) (except the momentum $k=$ $\pi, 2 \pi)$. The imaginary part of the energy spectrum is enlarged as the degree of coupling asymmetry (imaginary magnetic flux) increases. Under open boundary condition, the degree of asymmetry in the coupling does not influence the spectrum and the spectrum is entirely real.
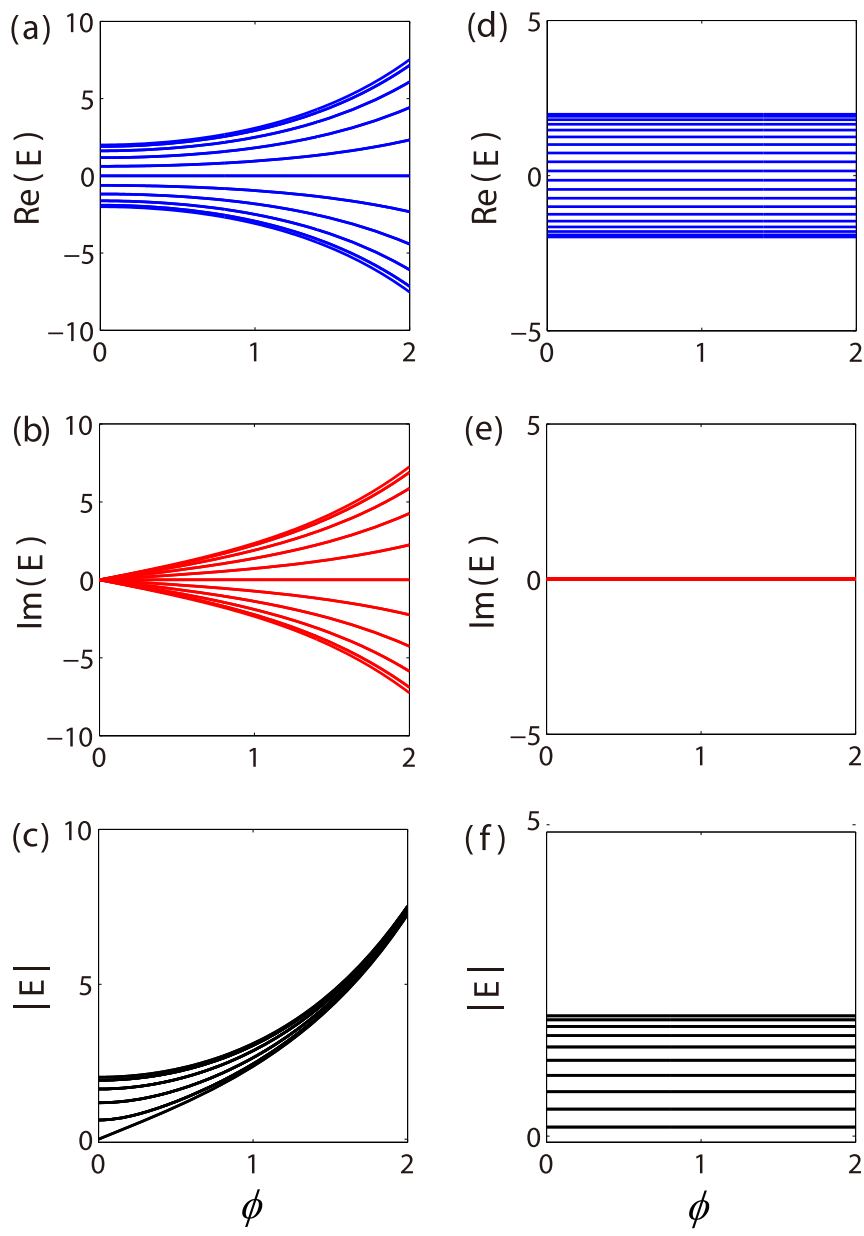

FIG. 2. Complex energy spectra of the unform coupled nonHermitian lattice with asymmetric coupling. (a) Real, (b) imaginary, and (c) absolute values of the spectrum in the closed ring configuration. (d) Real, (e) imaginary, and (f) absolute values of the spectrum in the open chain configuration. The system size is $N=20, \alpha \beta=1$.

\section{NONUNIFORM SYSTEM}

The non-Hermitian asymmetric coupling does not inevitably induce the non-Hermitian phase transition. In this section, we discuss a uniform non-Hermitian system with single different coupling between the head and tail of the periodical system. The Hamiltonian reads

$$
\begin{aligned}
H_{\mathrm{Non}}= & \sqrt{\alpha \beta}\left[\sum_{j=1}^{N-1}\left(e^{\phi} a_{j}^{\dagger} a_{j+1}+e^{-\phi} a_{j+1}^{\dagger} a_{j}\right)\right. \\
& \left.+J e^{\phi} a_{N}^{\dagger} a_{1}+J e^{-\phi} a_{1}^{\dagger} a_{N}\right],
\end{aligned}
$$

where $e^{\phi}=\sqrt{\alpha / \beta}$ and $J$ is an enlarged or diminished amplitude, indicating the strength difference between the normal uniform coupling and the abnormal coupling. Considering $H_{\text {Non }}$ in a gauge field, the energy spectrum of $H=$ $\sqrt{\alpha \beta}\left[\sum_{j=1}^{N-1}\left(\bar{b}_{j} b_{j+1}+\bar{b}_{j+1} b_{j}\right)+J e^{N \phi} \bar{b}_{N} b_{1}+J e^{-N \phi} \bar{b}_{1} b_{N}\right]$ is studied. The dispersion relation is still in the form of $E_{n}=$ $2 \sqrt{\alpha \beta} \cos k_{n}$. From the Schrödinger equations, we obtain the 
critical equation for the momentum $k_{n}$, which is a transcendental equation in the form of

$2 J \cosh (\phi N) \sin k_{n}=\sin \left[k_{n}(1+N)\right]+J^{2} \sin \left[k_{n}(1-N)\right]$.

We set $k_{n}=2 n \pi / N+\theta_{n}$. For a large $N, N \theta_{n}$ is nonzero finite and $\theta_{n} \approx 0$. Equation (8) reduces to

$$
\sin \left(\eta_{n}+\theta_{n} N\right)=2 J \cosh (\phi N) \sin (2 n \pi / N) / \xi_{n}
$$

(except for $n=N / 2, N)$, where $\eta_{n}$ satisfies

$$
\begin{aligned}
& \sin \eta_{n}=\left(1+J^{2}\right) \sin (2 n \pi / N) / \xi_{n}, \\
& \cos \eta_{n}=\left(1-J^{2}\right) \cos (2 n \pi / N) / \xi_{n},
\end{aligned}
$$

and

$$
\xi_{n}=\sqrt{\left(1-J^{2}\right)^{2}+4 J^{2} \sin ^{2}(2 n \pi / N)} .
$$

Therefore, the condition of the spectrum with a complex energy level is the occurrence of complex $\theta_{n}$, which indicates the non-Hermitian phase transition [6] and requires $\left|\sin \left(\eta_{n}+\theta_{n} N\right)\right|>1$. The condition for the energy level $n$ to be complex is given by

$$
\sinh ^{2}(\phi N)>\frac{\left(1-J^{2}\right)^{2}}{4 J^{2} \sin ^{2}(2 n \pi / N)},
$$

where the most fragile energy level is the one with $2 n \pi / N$ closest to $\pi / 2$. For fixed $\phi$, we take system size $N=4 n$ as an illustration, where $2 n \pi / N=\pi / 2$ at $n=N / 4$ and the critical coupling amplitude $J_{c}$ for exceptional points [11] is

$$
J_{c}= \pm e^{ \pm \phi N} .
$$

The real and imaginary parts of the energy levels at the parameter $\sqrt{\alpha / \beta}=1.05$ are depicted in Figs. 3(a) and 3(b). In Fig. 3(a), as $J$ increases, two energy levels are out of the energy bands, forming the bound states. In Fig. 3(b), we notice four critical $J_{c}$; the regions with zero imaginary part inside the central two $J_{c}$ and outside the outer two $J_{c}$ are in phase with the entirely real spectrum. At different $J$, the number of complex energy levels differs, which can be seen in Fig. 3(b). Considering Eq. (8) in the case of odd $N$, the spectrum of a system with $-J$ possesses $\pi$ shift of momentum $(k \rightarrow k+\pi)$ for a system with $J$; thus, the spectrum of a system with $-J$ is inversed $\left(E_{k} \rightarrow-E_{k}\right)$ in comparison to a system with $J$. This conclusion is not valid for even $N$. The number of complex energy levels differs at $J= \pm 1$, which is attributed to the energy level $n=N$ or $N / 2$. In the case of $n=N$ or $N / 2$, Eq. (8) reduces to

$$
\begin{aligned}
2 J \cosh (\phi N) \sin \theta_{n}= & \left(1+J^{2}\right) \sin \theta_{n} \cos \left(\theta_{n} N\right) \\
& +\left(1-J^{2}\right) \cos \theta_{n} \sin \left(\theta_{n} N\right) .
\end{aligned}
$$

For an odd $N$ and $n=N$, Eq. (14) reduces to $\cos \left(\theta_{n} N\right)=$ $\cosh (\phi N)$ at $J=1$, and obviously $\theta_{n}=i \phi$; at $J=-1$, we have $\theta_{n}=\pi+i \phi$. This indicates that one real energy level exists for odd $N$ when $|J|=1$. For even $N$ and $n=N / 2$ or $N$, $\theta_{n}=i \phi$ for $J=1$ only. For $J=-1$, Eq. (14) reduces to $\cos \left(\theta_{n} N\right)=-\cosh (\phi N)$, and one can rewrite it as

$$
\cos \left[N \theta_{n}+(N-1) \pi\right]=\cosh (\phi N),
$$
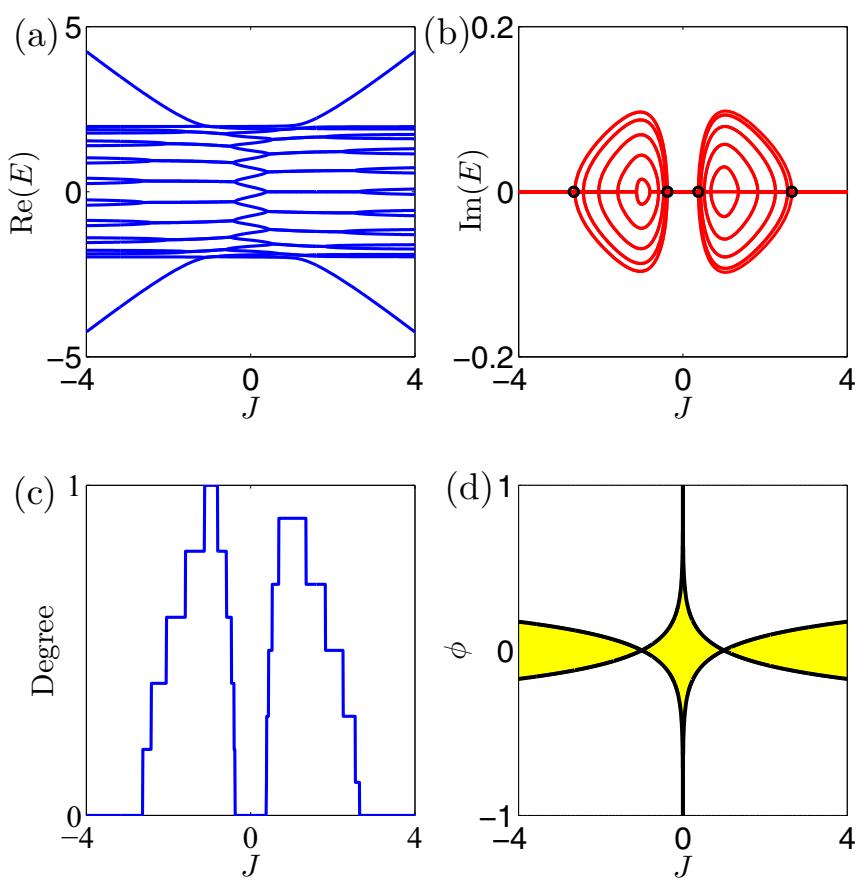

FIG. 3. (a) Real and (b) imaginary parts of the energy spectrum of a nonuniform non-Hermitian system with single different coupling. The black circles are $J_{c}$ from Eq. (13). (c) Degree of complex energy levels in the spectrum. (d) Phase diagram on the parameter $J-\phi$ plane. The yellow area indicates the entirely real spectrum, while the white area indicates the complex spectrum. The system size is $N=20$, and the parameter is $\sqrt{\alpha / \beta}=1.05$ in (a), (b), and (c).

where we can obtain $\theta_{n}=i \phi+(N-1) \pi / N$. Thus, all the energy levels are complex at $J=-1$, and two real energy levels exist at $J=1$ [Fig. 3(b)].

We define the number of complex energy levels over the total number of energy levels as the degree of complex energy levels [90], which is depicted as a function of $J$ in Fig. 3(c). The degree of complex energy levels is depicted as the signature of the plateau, which is a result of the finite-size effect. The plateau turns steep when $J$ approaches $J_{c}$ in the middle because the imaginary parts of the energy levels become sensitive as depicted in Fig. 3(b). The phase diagram for nonHermitian phase transition is depicted in Fig. 3(d). The yellow areas are regions with entirely real spectra; the white areas are regions with complex energy levels. The non-Hermitian phase transition occurs at the black curves. At $J=4$ in the yellow region, the system lies in the phase with the entirely real spectrum in spite of the fact that nonzero imaginary magnetic flux is present. At $J=1$, any nonzero imaginary magnetic flux leads to the non-Hermitian phase transition. This reflects that the imaginary magnetic flux does not inevitably induce the non-Hermitian phase transition for the nonuniform lattice with a single coupling defect.

\section{EIGENSTATES IN AN OPEN SYSTEM}

The imaginary gauge field does not influence the system energy spectrum under open boundary condition at $J=0$ of Hamiltonian Eq. (7), where the spectrum is entirely real. Although the eigenspectrum is unchanged under open boundary 
condition as depicted in Figs. 2(d)-2(f), the imaginary gauge field induces all the eigenstates localized at one system boundary. The scaling law of the localization effect only depends on the strength of the imaginary gauge field. The localization is a one-way amplification that is robust to disorder.

Under open boundary condition, the Hamiltonian reads

$$
H_{\text {chain }}=\sqrt{\alpha \beta} \sum_{j=1}^{N-1}\left(e^{\phi} a_{j}^{\dagger} a_{j+1}+e^{-\phi} a_{j+1}^{\dagger} a_{j}\right) .
$$

The coupling between the head and tail is absent.

We assume the eigenstates as

$$
\left|\psi_{n}^{\mathrm{R}}\right\rangle=\frac{1}{\Omega_{n}} \sum_{j=1}^{N} f_{j}^{n} a_{j}^{\dagger}|\mathrm{vac}\rangle,
$$

where the amplitude $f_{j}^{n}$ of the eigenstate is in the form of

$$
f_{j}^{n}=e^{-\phi j} \sin \left(k_{n} j\right),(j=1,2, \ldots, N),
$$

and the renormalization factor is

$$
\Omega_{n}=\sqrt{\sum_{j=1}^{N} e^{-2 j \phi} \sin ^{2}\left(k_{n} j\right) .}
$$

The detailed calculation is given in the Appendix. The Dirac norm of the eigenstates is defined as $\left\langle\psi_{n}^{\mathrm{R}} \mid \psi_{n}^{\mathrm{R}}\right\rangle$; notice that $\left\langle\psi_{n}^{\mathrm{R}} \mid \psi_{n}^{\mathrm{R}}\right\rangle=1$ for the right eigenstate $\left|\psi_{n}^{\mathrm{R}}\right\rangle$ with the renormalization factor $\Omega_{n}$. The eigenstates are not orthogonal in the non-Hermitian system [24]; thus the time evolution is nonunitary. The scalar light wave function in an optical system under paraxial approximation is analogous to the Schrödinger equation that characterizes the particle dynamics [91]. The light intensity is a physical observable, being the Dirac norm of the wave. The light intensity oscillates in the exact $\mathcal{P} \mathcal{T}$ symmetric phase, known as the power oscillation [26].

In Figs. 4(a) and 4(b), we depict the Dirac norm probability distribution of typical eigenstates in a system with size $N=$ 40 , where all the eigenstates are localized at one boundary of the non-Hermitian lattice, known as the non-Hermitian skin effect [82-89]. The skin effect is due to the directional amplification or attenuation of the asymmetric coupling, thus eigenstate amplitude is one-way amplified $(\alpha>\beta)$.

To investigate the localization effect induced by the asymmetric coupling, the IPR is studied. The IPR for eigenstate $\left|\psi_{n}^{\mathrm{R}}\right\rangle$ is defined as

$$
\chi_{n}=\frac{\sum_{j=1}^{N}\left|f_{j}^{n}\right|^{4}}{\left(\sum_{j=1}^{N}\left|f_{j}^{n}\right|^{2}\right)^{2}} .
$$

The IPR is valuable in capturing the localization properties of eigenstates. The IPR of the localization states is insensitive to the system size; in contrast, the IPR of the extended states is inversely proportional to the system size. We employ the averaged IPR to reveal the localization properties of the eigenstates, $\chi=(1 / N) \sum_{n=1}^{N} \chi_{n}$ :

$$
\chi=\frac{1}{N} \sum_{n=1}^{N} \frac{1}{\Omega_{n}^{4}} \sum_{j=1}^{N} e^{-4 \phi j} \sin ^{4}\left(k_{n} j\right) .
$$

(a)

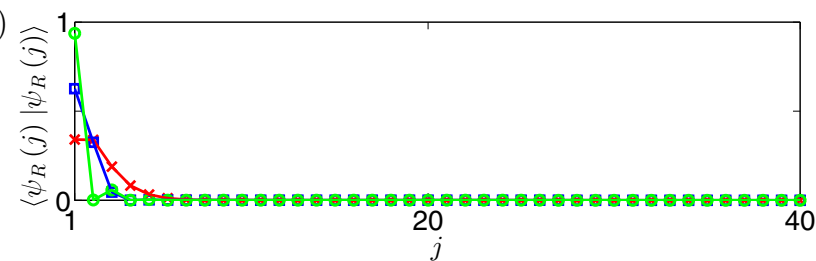

(b)
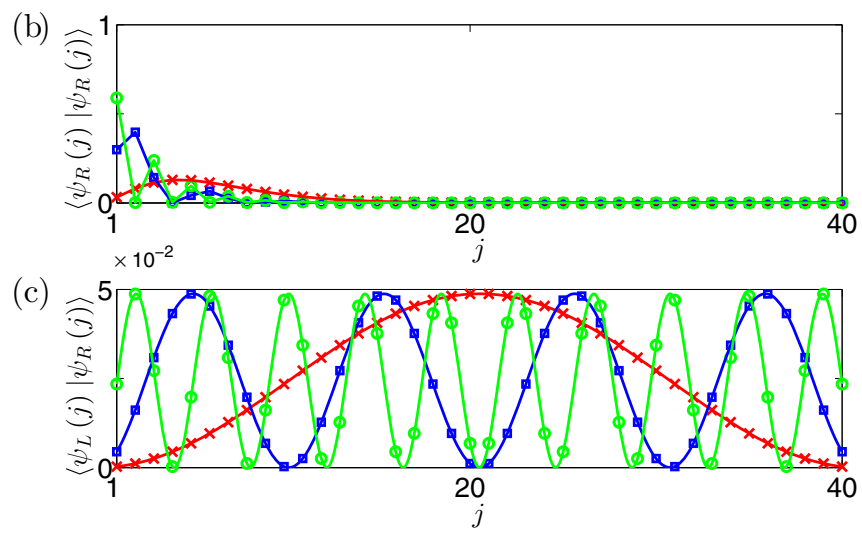

FIG. 4. The eigenstates of the non-Hermitian lattice with asymmetric coupling. We select three eigenstates in the open chain system at a certain asymmetric coupling. The eigenstates are localized at the lattice boundary in (a) and (b) and are extended in (c). The parameter is $\sqrt{\alpha / \beta}=2$ in (a) and (c) and $\sqrt{\alpha / \beta}=5 / 4$ in (b).

We can obtain the asymptotic value of the averaged IPR at the limit $N \rightarrow \infty$ in the form of

$$
\chi_{c}=\frac{1}{4} \tanh \phi\left(\operatorname{sech}^{4} \phi \tanh \phi-8 \tanh \phi+6 \tanh ^{2} \phi+6\right),
$$

which only depends on the asymmetric coupling strength.

In Fig. 5, we depict the averaged IPR as a function of the system size and asymmetric coupling strength. The asymptotic values of the averaged IPR [Eq. (22)] at different asymmetric couplings are indicated by the dotted black lines. The scaling behavior of the averaged IPR reflects the localization effect of the eigenstates. At small system size, the slope of IPR approaches -1 ; the exponential decay of the eigenstates and the localization effect are not obvious to be observed at weak-coupling asymmetry. Therefore, the eigenstates exhibit extended behavior, being inversely proportional to the system size. At large system size or at large coupling asymmetry, the slope of the IPR in Fig. 5 goes to zero; the localization effect is obvious and the averaged IPR becomes insensitive to the system size. At significantly strong asymmetry, the eigenstates are tightly localized at several sites near the system boundary (solid black line). The inset of Fig. 5 depicts the relation between the asymptotic values of the averaged IPR and the asymmetric coupling; the system size is chosen as $N=40 ; \chi_{c}$ is approximately proportional to $1-\sqrt{\alpha / \beta}$. The localization length of the eigenstates is $[\ln (\sqrt{\alpha / \beta})]^{-1}[92]$.

\section{BIORTHOGONAL IPR}

The real gauge field does not influence an open system; although the Dirac norm of eigenstate distribution and the IPR are affected by the asymmetric coupling, the gauge 


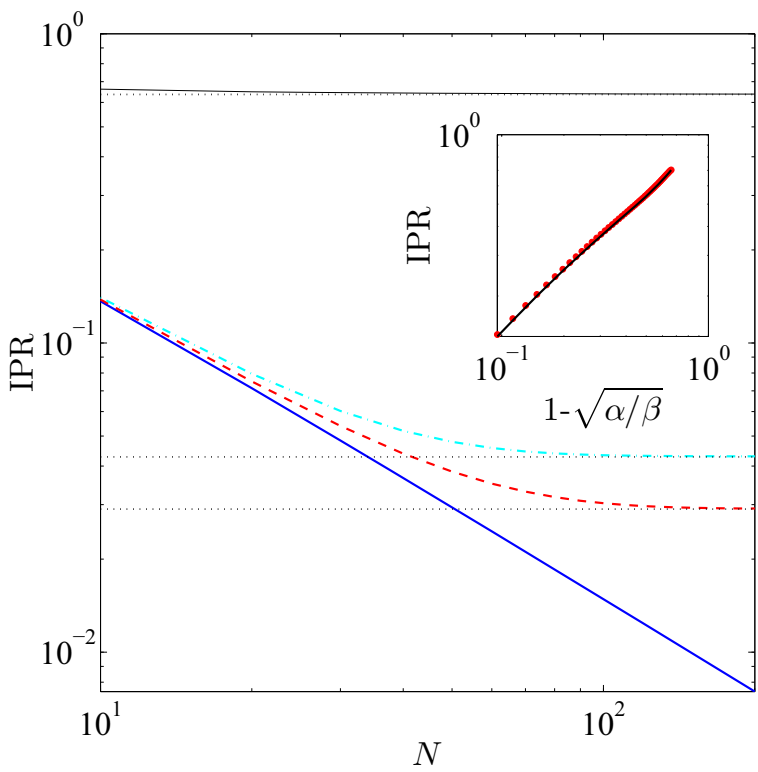

FIG. 5. The averaged IPR of the eigenstates. The curves are for different degrees of asymmetry. Solid black curve, $\sqrt{\alpha / \beta}=2.5$; dash-dotted green curve, $\sqrt{\alpha / \beta}=1.03$; dashed red curve, $\sqrt{\alpha / \beta}=$ 1.02; dotted blue curve, uniform ring with symmetric coupling for comparison. The asymptotic value at large $N$ is indicated by the dotted black lines. Inset: The averaged IPR as a function of coupling asymmetry at $N=40$. The red points are obtained through numerical simulation and the black lines are plotted from Eq. (22).

invariant properties of the imaginary field are reflected from the biorthogonal basis. To better understand the influence of asymmetric coupling and the imaginary gauge field, we investigate the biorthogonal norm of the eigenstates. According to the right eigenstates in Eq. (17), the left eigenstates of the uniform chain satisfy the Schrödinger equation $H_{\text {chain }}^{\dagger}\left|\psi_{n}^{\mathrm{L}}\right\rangle=\varepsilon_{n}^{*}\left|\psi_{n}^{\mathrm{L}}\right\rangle$. The left eigenstates are given by $\left|\psi_{n}^{\mathrm{L}}\right\rangle=\left(\Omega_{n} / \Lambda_{n}\right) \sum_{j=1}^{N} \tilde{f}_{j}^{n} a_{j}^{\dagger}|\mathrm{vac}\rangle$ with

$$
\tilde{f}_{j}^{n}=e^{\phi j} \sin \left(k_{n} j\right), \Lambda_{n}=\sum_{j=1}^{N}\left|\tilde{f}_{j}^{n} f_{j}^{n}\right|,
$$

and the left and right eigenstates satisfy the biorthogonal relation

$$
\left\langle\psi_{m}^{\mathrm{L}} \mid \psi_{n}^{\mathrm{R}}\right\rangle=\delta_{m n},
$$

for any integer $m, n \in[1, N]$. The biorthogonal norm of the right eigenstates $\left|\psi_{n}^{\mathrm{R}}\right\rangle$ is defined as $\left\langle\psi_{n}^{\mathrm{L}} \mid \psi_{n}^{\mathrm{R}}\right\rangle$. Typical eigenstate distribution under the biorthogonal norm is plotted in Fig. 4(c), where we observe the extended states in the form of sinusoidal functions.

Under the biorthogonal norm, the imaginary gauge field has no influence in the system under open boundary condition; in contrast, under the Dirac norm, the real gauge field has no influence in the system under open boundary condition. This reflects the gauge invariant properties of the imaginary gauge field induced by the asymmetric coupling. The biorthogonal IPR, defined from both the left and right eigenstates, is given by

$$
\tilde{\chi}_{n}=\frac{\sum_{j=1}^{N}\left|\tilde{f}_{j}^{n} f_{j}^{n}\right|^{2}}{\left(\sum_{j=1}^{N}\left|\tilde{f}_{j}^{n} f_{j}^{n}\right|\right)^{2}} .
$$

Correspondingly the averaged biorthogonal IPR is given by $\tilde{\chi}=(1 / N) \sum_{n=1}^{N} \tilde{\chi}_{n}$. Through a direct simplification after substituting the expressions of $f_{j}^{n}$ and $\tilde{f}_{j}^{n}$, we obtain the averaged biorthogonal IPR as

$$
\tilde{\chi}=\frac{3}{2(N+1)} .
$$

The averaged biorthogonal IPR is inversely proportional to the system size, which implies that the eigenstate is extended and reveals that the asymmetric coupling induces a gauge field under the biorthogonal norm. In contrast, the intensity localization under the Dirac norm indicates the one-way amplification or attenuation. The average IPR efficiently evaluates the localization of eigenstates. The proposed asymmetric coupling provides a route toward nonreciprocal phenomena in photonics, which are valuable for unidirectional light modulation and optical isolation.

\section{CONCLUSION}

In summary, we investigate a uniform non-Hermitian lattice with asymmetric coupling. The asymmetric coupling induces an imaginary magnetic flux enclosed in a closed ring configuration, which directly leads to the non-Hermitian phase transition in which all the eigenvalues become complex except for the special momentum $k=\pi, 2 \pi$; however, for the system with nonuniform coupling, the imaginary magnetic flux does not inevitably induce the non-Hermitian phase transition. Under open boundary condition, the eigenvalues are not influenced by the asymmetric coupling; however, the eigenstates are localized at the system boundary due to the amplification or attenuation effect of the asymmetric coupling. The scaling law for the eigenstates is shown by employing the averaged IPR. The left eigenstates and biorthogonal norm are studied. The biorthogonal probability distribution of eigenstates is a sinusoidal function, being extended. The averaged biorthogonal IPR defined from both left and right eigenstates is inversely proportional to the system size. Our findings reflect the underlying physics induced by the asymmetric coupling and are valuable for further investigations of nonreciprocity and non-Hermitian phase transition in photonics and beyond.

\section{ACKNOWLEDGMENTS}

We acknowledge support by National Natural Science Foundation of China (Grants No. 11605094 and No. 11874225) and by the Fundamental Research Funds for the Central Universities, Nankai University (Grants No. 63191522 and No. 63191738).

\section{APPENDIX}

Here, we show the method of obtaining the eigenstate of the system in detail. The Schrödinger equations of the 
Hamiltonian $H\left|\psi_{n}^{\mathrm{R}}\right\rangle=\sqrt{\alpha \beta} \varepsilon_{n}\left|\psi_{n}^{\mathrm{R}}\right\rangle$ yield a set of linear equations:

$$
f_{j+1}^{n}-e^{-\phi} \varepsilon_{n} f_{j}^{n}+e^{-2 \phi} f_{j-1}^{n}=0
$$

for $2<j<N-1$; at the lattice boundary, we have two other equations:

$$
\begin{array}{r}
-\varepsilon_{n} f_{1}^{n}+e^{\phi} f_{2}^{n}=0, \\
-\varepsilon_{n} f_{N}^{n}+e^{-\phi} f_{N-1}^{n}=0 .
\end{array}
$$

We rewrite the Schrödinger equation $f_{j+1}^{n}-e^{-\phi} \varepsilon_{n} f_{j}^{n}+$ $e^{-2 \phi} f_{j-1}^{n}=0$ as

$$
f_{j+1}^{n}-\mu f_{j}^{n}=\lambda\left(f_{j}^{n}-\mu f_{j-1}^{n}\right),
$$

where $j=2, \ldots, N-1$, and $\left\{f_{j}^{n}-\mu f_{j-1}^{n}\right\}$ is a nongeometric series. Comparing the coefficients with the original Schrödinger equation, we obtain

$$
\begin{aligned}
\mu+\lambda & =e^{-\phi} \varepsilon_{n}, \\
\lambda \mu & =e^{-2 \phi},
\end{aligned}
$$

where $\mu$ and $\lambda$ are the roots of the above equations:

$$
\mu, \lambda=\frac{\varepsilon_{n} \pm \sqrt{\varepsilon_{n}^{2}-4}}{2 e^{\phi}} .
$$

According to Eqs. (A3) and (A5), we get

$$
\begin{gathered}
f_{j}^{n}-\mu f_{j-1}^{n}=\lambda^{j-2}\left(f_{2}^{n}-\mu f_{1}^{n}\right), \\
f_{j}^{n}-\lambda f_{j-1}^{n}=\mu^{j-2}\left(f_{2}^{n}-\lambda f_{1}^{n}\right) .
\end{gathered}
$$

Substituting the second line into the first line we obtain the expression

$$
f_{j}^{n}=\frac{\left(f_{2}^{n}-\mu f_{1}^{n}\right) \lambda^{j-1}-\left(f_{2}^{n}-\lambda f_{1}^{n}\right) \mu^{j-1}}{\lambda-\mu} .
$$

Assuming $f_{1}^{n}=1$, based on one boundary condition $\varepsilon_{n} e^{-\phi} f_{1}^{n}=f_{2}^{n}$ and the first line of Eq. (A4), Eq. (A7) reduces to

$$
f_{j}^{n}=\frac{\lambda^{j}-\mu^{j}}{\lambda-\mu}
$$

After substituting $f_{j}^{n}$ into anther boundary condition $\varepsilon_{n} f_{N}^{n}=$ $e^{-\phi} f_{N-1}^{n}$, employing Eq. (A4), we obtain

$$
\left(\lambda e^{\phi}\right)^{2(N+1)}=1
$$

which means

$$
\lambda e^{\phi}=e^{i k_{n}}
$$

for $k_{n}=n \pi /(N+1)$ with $n=1, \ldots, N$. From Eq. (A5), we obtain $\varepsilon_{n}+\sqrt{\varepsilon_{n}^{2}-4}=2 e^{i k_{n}}$ or $\varepsilon_{n}-\sqrt{\varepsilon_{n}^{2}-4}=2 e^{i k_{n}}$. In any case, the energy is given by

$$
\varepsilon_{n}=2 \cos k_{n}
$$

Substituting $\varepsilon_{n}$ into $f_{j}^{n}$ and considering the normalization factor, we obtain the wave functions

$$
f_{j}^{n}=e^{-\phi j} \sin \left(k_{n} j\right),
$$

where $j=1, \ldots, N$. The Dirac normalization factor is

$$
\Omega_{n}=\sqrt{\sum_{j=1}^{N} e^{-2 j \phi} \sin ^{2}\left(k_{n} j\right)} .
$$

[1] C. M. Bender, Rep. Prog. Phys. 70, 947 (2007).

[2] N. Moiseyev, Non-Hermitian Quantum Mechanics (Cambridge University, Cambridge, England, 2011).

[3] L. Feng, R. El-Ganainy, and L. Ge, Nat. Photo. 11, 752 (2017).

[4] S. Longhi, Eur. Phys. Lett. 120, 64001 (2017).

[5] Y. Huang, Y. Shen, C. Min, S. Fan, and G. Veronis, Nanophotonics 6, 977 (2017).

[6] R. El-Ganainy, K. G. Makris, M. Khajavikhan, Z. H. Musslimani, S. Rotter, and D. N. Christodoulides, Nat. Phys. 14, 11 (2018).

[7] S. K. Gupta, Y. Zou, X. Y. Zhu, M. H. Lu, L. Zhang, X. P. Liu, and Y. F. Chen, arXiv:1803.00794.

[8] M. A. Miri and A. Alù, Science 363, eaar7709 (2019).

[9] A. Guo, G. J. Salamo, D. Duchesne, R. Morandotti, M. VolatierRavat, V. Aimez, G. A. Siviloglou, and D. N. Christodoulides, Phys. Rev. Lett. 103, 093902 (2009).

[10] C. Dembowski, B. Dietz, H. D. Gräf, H. L. Harney, A. Heine, W. D. Heiss, and A. Richter, Phys. Rev. E 69, 056216 (2004); M. M üler and I. Rotter, J. Phys. A 41, 244018 (2008); R. Uzdin, A. Mailybaev, and N. Moiseyev, ibid. 44, 435302 (2011).

[11] W. D. Heiss, J. Phys. A 45, 444016 (2012).
[12] B. Zhen, C. W. Hsu, Y. Igarashi, L. Lu, I. Kaminer, A. Pick, S. L. Chua, J. D. Joannopoulos, and M. Soljačić, Nature (London) 525, 354 (2015).

[13] J. Doppler, A. A. Mailybaev, J. Böhm, U. Kuhl, A. Girschik, F. Libisch, T. J. Milburn, P. Rabl, N. Moiseyev, and S. Rotter, Nature (London) 537, 76 (2016).

[14] H. Xu, D. Mason, L. Jiang, and J. G. E. Harris, Nature (London) 537, 80 (2016).

[15] K. Ding, G. Ma, M. Xiao, Z. Q. Zhang, and C. T. Chan, Phys. Rev. X 6, 021007 (2016); X. L. Zhang, S. B. Wang, B. Hou, and C. T. Chan, ibid. 8, 021066 (2018).

[16] S. Assawaworrarit, X. Yu, and S. Fan, Nature (London) 546, 387 (2017).

[17] H. Jing, S. K. Ozdemir, H. Lu, and F. Nori, Sci. Rep. 7, 3386 (2017).

[18] L. Jin, Phys. Rev. A 97, 012121 (2018).

[19] L. Pan, S. Chen, and X. Cui, Phys. Rev. A 99, 011601(R) (2019).

[20] P. Wang, L. Jin, G. Zhang, and Z. Song, Phys. Rev. A 94, 053834 (2016); Q. Zhong, D. N. Christodoulides, M. Khajavikhan, K. G. Makris, and R. El-Ganainy, ibid. 97, 020105(R) (2018); L. Ge, Photon. Res. 6, A10 (2018). 
[21] M. Am-Shallem, R. Kosloff, and N. Moiseyev, New J. Phys. 17, 113036 (2015).

[22] J. Wiersig, Phys. Rev. Lett. 112, 203901 (2014); W. Chen, S. K. Ozdemir, G. Zhao, J. Wiersig, and L. Yang, Nature (London) 548, 192 (2017); H. Hodaei, A. U. Hassan, S. Wittek, H. Garcia-Gracia, R. El-Ganainy, D. N. Christodoulides, and M. Khajavikhan, ibid. 548, 187 (2017).

[23] Z. P. Liu, J. Zhang, Ş. K. Ödemir, B. Peng, H. Jing, X. Y. Lü, C. W. Li, L. Yang, F. Nori, and Y. X. Liu, Phys. Rev. Lett. 117, 110802 (2016).

[24] K. G. Makris, R. El-Ganainy, D. N. Christodoulides, and Z. H. Musslimani, Phys. Rev. Lett. 100, 103904 (2008).

[25] S. Klaiman, U. Gunther, and N. Moiseyev, Phys. Rev. Lett. 101, 080402 (2008).

[26] C. E. Rüter, K. G. Makris, R. El-Ganainy, D. N. Christodoulides, M. Segev, and D. Kip, Nat. Phys. 6, 192 (2010).

[27] Y. D. Chong, L. Ge, H. Cao, and A. D. Stone, Phys. Rev. Lett. 105, 053901 (2010); W. Wan, Y. Chong, L. Ge, H. Noh, A. D. Stone, and H. Cao, Science 331, 889 (2011).

[28] A. Regensburger, C. Bersch, M. A. Miri, G. Onishchukov, D. N. Christodoulides, and U. Peschel, Nature (London) 488, 167 (2012).

[29] L. Feng, Y. L. Xu, W. S. Fegadolli, M. H. Lu, José. E. B. Oliveira, V. R. Almeida, Y. F. Chen, and A. Scherer, Nat. Mater. 12, 108 (2013).

[30] H. Ramezani, Y. Wang, E. Yablonovitch, and X. Zhang, Ieee. J. Sel. Top. Quant. 22, 5000706 (2016); L. Jin, P. Wang, and Z. Song, Sci. Rep. 6, 32919 (2016).

[31] L. Jin and Z. Song, Phys. Rev. Lett. 121, 073901 (2018).

[32] H. Ramezani, H. K. Li, Y. Wang, and X. Zhang, Phys. Rev. Lett. 113, 263905 (2014).

[33] B. Peng, S. K. Özdemir, M. Liertzer, W. J. Chen, J. Kramer, H. Yilmaz, J. Wiersig, S. Rotter, and L. Yang, Proc. Natl. Acad. Sci. USA 113, 6845 (2016).

[34] Y. Kominis, T. Bountis, and S. Flach, Sci. Rep. 6, 33699 (2016); Phys. Rev. A 95, 063832 (2017); Y. Kominis, K. D. Choquette, T. Bountis, and V. Kovanis, Appl. Phys. Lett. 113, 081103 (2018).

[35] J. H. Wu, M. Artoni, and G. C. La Rocca, Phys. Rev. Lett. 113, 123004 (2014); Z. Zhang, Y. Zhang, J. Sheng, L. Yang, M. A. Miri, D. N. Christodoulides, B. He, Y. Zhang, and M. Xiao, ibid. 117, 123601 (2016); P. Peng, W. Cao, C. Shen, W. Qu, J. Wen, L. Jiang, and Y. Xiao, Nat. Phys. 12, 1139 (2016).

[36] A. Ruschhaupt, F. Delgado, and J. G. Muga, J. Phys. A 38, L171 (2005).

[37] R. El-Ganainy, K. G. Makris, D. N. Christodoulides, and Z. H. Musslimani, Opt. Lett. 32, 2632 (2007).

[38] Y. L. Xu, W. S. Fegadolli, L. Gan, M. H. Lu, X. P. Liu, Z. Y. Li, A. Scherer, and Y. F. Chen, Nat. Commun. 7, 11319 (2016).

[39] B. Peng, S. K. Özdemir, F. Lei, F. Monifi, M. Gianfreda, G. L. Long, S. Fan, F. Nori, C. M. Bender, and L. Yang, Nat. Phys. 10, 394 (2014).

[40] H. Jing, S. K. Ozdemir, X. Y. Lu, J. Zhang, L. Yang, and F. Nori, Phys. Rev. Lett. 113, 053604 (2014).

[41] B. He, L. Yang, and M. Xiao, Phys. Rev. A 94, 031802(R) (2016); Phys. Rev. Lett. 120, 203904 (2018).

[42] A. Cerjan, A. Raman, and S. Fan, Phys. Rev. Lett. 116, 203902 (2016).
[43] C. He, X. C. Sun, X. P. Liu, M. H. Lu, Y. Chen, L. Feng, and Y. F. Chen, Proc. Natl. Acad. Sci. USA 113, 4924 (2016).

[44] J. Schindler, A. Li, M. C. Zheng, F. M. Ellis, and T. Kottos, Phys. Rev. A 84, 040101(R) (2011); J. Schindler, Z. Lin, J. M. Lee, H. Ramezani, F. M. Ellis, and T. Kottos, J. Phys. A 45, 444029 (2012).

[45] R. Fleury, D. Sounas, and A. Alù, Nat. Commun. 6, 5905 (2015); S. A. Cummer, J. Christensen, and A. Alù, Nat. Rev. Mater. 1, 16001 (2016).

[46] X. Zhu, H. Ramezani, C. Shi, J. Zhu, and X. Zhang, Phys. Rev. X 4, 031042 (2014).

[47] L. Chang, X. Jiang, S. Hua, C. Yang, J. Wen, L. Jiang, G. Li, G. Wang, and M. Xiao, Nat. Photon. 8, 524 (2014).

[48] L. Feng, Z. J. Wong, R. M. Ma, Y. Wang, and X. Zhang, Science 346, 972 (2014).

[49] G. Harari, M. A. Bandres, Y. Lumer, M. C. Rechtsman, Y. D. Chong, M. Khajavikhan, D. N. Christodoulides, and M. Segev, Science 359, eaar4003 (2018); M. A. Bandres, S. Wittek, G. Harari, M. Parto, J. Ren, M. Segev, D. Christodoulides, and M. Khajavikhan, ibid. 359, eaar4005 (2018).

[50] S. Longhi, D. Gatti, and G. Della Valle, Sci. Rep. 5, 13376 (2015); Phys. Rev. B 92, 094204 (2015).

[51] B. Midya, H. Zhao, and L. Feng, Nat. Commun. 9, 2674 (2018).

[52] Z. P. Gong, Y. Ashida, K. Kawabata, K. Takasan, S. Higashikawa, and M. Ueda, Phys. Rev. X 8, 031079 (2018).

[53] L. Jin, Phys. Rev. A 96, 032103 (2017); L. Jin, P. Wang, and Z. Song, Sci. Rep. 7, 5903 (2017).

[54] S. Weimann, M. Kremer, Y. Plotnik, Y. Lumer, S. Nolte, K. G. Makris, M. Segev, M. C. Rechtsman, and A. Szameit, Nat. Mater. 16, 433 (2017).

[55] J. M. Zeuner, M. C. Rechtsman, Y. Plotnik, Y. Lumer, S. Nolte, M. S. Rudner, M. Segev, and A. Szameit, Phys. Rev. Lett. 115, 040402 (2015).

[56] H. Schomerus, Opt. Lett. 38, 1912 (2013); S. Malzard, C. Poli, and H. Schomerus, Phys. Rev. Lett. 115, 200402 (2015); C. Poli, M. Bellec, U. Kuhl, F. Mortessagne, and H. Schomerus, Nat. Commun. 6, 6710 (2015).

[57] H. Zhao, S. Longhi, and L. Feng, Sci. Rep. 5, 17022 (2015); M. Pan, H. Zhao, P. Miao, S. Longhi, and L. Feng, Nat. Commun. 9, 1308 (2018); B. Midya and L. Feng, Phys. Rev. A 98, 043838 (2018).

[58] H. Zhao, P. Miao, M. H. Teimourpour, S. Malzard, R. ElGanainy, H. Schomerus, and L. Feng, Nat. Commun. 9, 981 (2018); M. Parto, S. Wittek, H. Hodaei, G. Harari, M. A. Bandres, J. Ren, M. C. Rechtsman, M. Segev, D. N. Christodoulides, and M. Khajavikhan, Phys. Rev. Lett. 120, 113901 (2018).

[59] G. Q. Liang and Y. D. Chong, Phys. Rev. Lett. 110, 203904 (2013); B. Zhu, R. Lü, and S. Chen, Phys. Rev. A 89, 062102 (2014); C. Yin, H. Jiang, L. Li, R. Lü, and S. Chen, ibid. 97, 052115 (2018); H. Jiang, C. Yang, and S. Chen, ibid. 98, 052116 (2018); C. H. Liu, H. Jiang, and S. Chen, Phys. Rev. B 99, 125103 (2019).

[60] S. Diehl, E. Rico, M. A. Baranov, and P. Zoller, Nat. Phys. 7, 971 (2011); Y. C. Hu and T. L. Hughes, Phys. Rev. B 84, 153101 (2011); K. Esaki, M. Sato, K. Hasebe, and M. Kohmoto, ibid. 84, 205128 (2011).

[61] J. Gong and Q. H. Wang, Phys. Rev. A 91, 042135 (2015); L. Zhou, Q. H. Wang, H. Wang, and J. Gong, ibid. 98, 022129 (2018). 
[62] D. Leykam, K. Y. Bliokh, C. Huang, Y. D. Chong, and F. Nori, Phys. Rev. Lett. 118, 040401 (2017); T. Liu, Y. R. Zhang, Q. Ai, Z. Gong, K. Kawabata, M. Ueda, and F. Nori, ibid. 122, 076801 (2019).

[63] Y. Xu, S. T. Wang, and L. M. Duan, Phys. Rev. Lett. 118, 045701 (2017); Q. B. Zeng, Y. B. Yang, and Y. Xu, arXiv:1901.08060.

[64] H. Shen, B. Zhen, and L. Fu, Phys. Rev. Lett. 120, 146402 (2018); H. Shen and L. Fu, ibid. 121, 026403 (2018).

[65] K. Yokomizo and S. Murakami, arXiv:1902.10958.

[66] C. Yuce, Phys. Lett. A 379, 1213 (2015); Phys. Rev. A 93, 062130 (2016); 97, 042118 (2018); 98, 012111 (2018); Z. Oztas and C. Yuce, ibid. 98, 042104 (2018); Z. Ozcakmakli Turker and C. Yuce, ibid. 99, 022127 (2019).

[67] S. Lieu, Phys. Rev. B 97, 045106 (2018); 98, 115135 (2018).

[68] F. Munoz, F. Pinilla, J. Mella, and M. I. Molina, Sci. Rep. 8, 17330 (2018).

[69] F. K. Kunst, E. Edvardsson, J. C. Budich, and E. J. Bergholtz, Phys. Rev. Lett. 121, 026808 (2018); J. Carlström and E. J. Bergholtz, Phys. Rev. A 98, 042114 (2018); E. Edvardsson, F. K. Kunst, and E. J. Bergholtz, Phys. Rev. B 99, 081302(R) (2019); J. Carlström, M. Stålhammar, J. C. Budich, and E. J. Bergholtz, ibid. 99, 161115(R) (2019); J. C. Budich, J. Carlström, F. K. Kunst, and E. J. Bergholtz, ibid. 99, 041406(R) (2019).

[70] K. Kawabata, K. Shiozaki, and M. Ueda, Phys. Rev. B 98, 165148 (2018); K. Kawabata, Y. Ashida, H. Katsura, and M. Ueda, ibid. 98, 085116 (2018); K. Kawabata, S. Higashikawa, Z. Gong, Y. Ashida, and M. Ueda, Nat. Commun. 10, 297 (2019); K. Kawabata, K. Shiozaki, M. Ueda, and M. Sato, arXiv:1812.09133.

[71] L. J. Lang, Y. Wang, H. Wang, and Y. D. Chong, Phys. Rev. B 98, 094307 (2018); X. Ni, D. Smirnova, A. Poddubny, D. Leykam, Y. Chong, and A. B. Khanikaev, ibid. 98, 165129 (2018).

[72] J. Hou, Z. Li, X. W. Luo, Q. Gu, and C. Zhang, arXiv:1808.06972.

[73] K. Takata and M. Notomi, Phys. Rev. Lett. 121, 213902 (2018).

[74] R. Okugawa and T. Yokoyama, Phys. Rev. B 99, 041202(R) (2019).
[75] A. Ghatak and T. Das, Phys. Rev. B 97, 014512 (2018); B. X. Wang and C. Y. Zhao, ibid. 98, 165435 (2018); Phys. Rev. A 98, 023808 (2018).

[76] K. Moors, A. A. Zyuzin, A. Y. Zyuzin, R. P. Tiwari, and T. L. Schmidt, Phys. Rev. B 99, 041116(R) (2019); V. M. Martinez Alvarez, J. E. Barrios Vargas, and L. E. F. Foa Torres, ibid. 97, 121401(R) (2018); V. M. Martinez Alvarez and M. D. Coutinho-Filho, Phys. Rev. A 99, 013833 (2019).

[77] H. Zhou and J. Y. Lee, Phys. Rev. B 99, 235112 (2019); F. K. Kunst and V. Dwivedi, arXiv:1812.02186.

[78] M. Ezawa, Phys. Rev. B 99, 201411(R) (2019); 99, 121411(R) (2019); arXiv:1902.03716; L. Herviou, J. H. Bardarson, and N. Regnault, arXiv:1901.0001; H. G. Zirnstein, G. Refael, and B. Rosenow, arXiv:1901.11241; M. R. Hirsbrunner, T. M. Philip, and M. J. Gilbert, arXiv:1901.09961.

[79] D. S. Borgnia, A. J. Kruchkov, and R. J. Slager, arXiv:1902.07217.

[80] V. M. Martinez Alvarez, J. E. Barrios Vargas, M. Berdakin, and L. E. F. Foa Torres, Eur. Phys. J. Spec. Top. 227, 1295 (2018).

[81] Z. Z. Li, X. S. Li, L. L. Zhang, and W. J. Gong, arXiv:1901.10688.

[82] S. Yao and Z. Wang, Phys. Rev. Lett. 121, 086803 (2018); S. Yao, F. Song, and Z. Wang, ibid. 121, 136802 (2018).

[83] Z. Yang and J. Hu, Phys. Rev. B 99, 081102(R) (2019).

[84] L. Jin and Z. Song, Phys. Rev. B 99, 081103(R) (2019).

[85] H. Wang, J. Ruan, and H. Zhang, Phys. Rev. B 99, 075130 (2019).

[86] C. H. Lee and R. Thomale, Phys. Rev. B 99, 201103(R) (2019); C. H. Lee, L. Li, and J. Gong, arXiv:1810.11824; C. H. Lee, G. Li, Y. Liu, T. Tai, R. Thomale, and X. Zhang, arXiv:1812.02011.

[87] K. Luo, J. Feng, Y. X. Zhao, and R. Yu, arXiv:1810.09231.

[88] H. Jiang, L. J. Lang, C. Yang, S. L. Zhu, and S. Chen, arXiv: 1901.09399.

[89] A. Ghatak and T. Das, J. Phys.: Condens. Matter 31, 263001 (2019).

[90] D. D. Scott and Y. N. Joglekar, Phys. Rev. A 83, 050102(R) (2011)

[91] S. Longhi, Laser Photo. Rev. 3, 243 (2009).

[92] L. Ge, Ann. Phys. (Berlin) 529, 1600182 (2017). 